\title{
A STATISTICAL ANALYSIS BETWEEN CMES AND FLARES OVER THE SOLAR
}

\section{CYCLE 24}

Eid A. Amin ${ }^{1}$, Abdelrazek M. K. Shaltout ${ }^{2}$, M. M. Beheary ${ }^{2}$, R. H. Hamid ${ }^{1}$

${ }^{I}$ Department of Solar and Space Research, National Research Institute of Astronomy and Geophysics (NRIAG), 11421 Helwan, Cairo, Egypt

${ }^{2}$ Department of Astronomy and Meteorology, Faculty of Science, Al-Azhar University, 11884 Nasr

City, Cairo, Egypt

\begin{abstract}
The correlations between the parameters of Coronal Mass Ejections (CMEs) and Solar Flares (SFs) during the solar cycle 24 are investigated. To do this, we first collect the CMEs and SFs sample over the period from December 1, 2008 to April 30, 2017. The CMEs data were obtained from the Solar and Heliospheric Observatory/ Large Angle Spectrometric Coronagraph (SOHO / LASCO) database while the soft X-ray flares data sets are taken from Hinode/XRT mission. Then, we compile a sample of 519 CMEs associated flares by applying temporal and spatial conditions. We statistically analyze our sample of CMEs associated flares and divide them into two types: after-CMEs and before-CMEs based on their start time. The CMEs and flares properties show that there were significant features in all physical properties such as (kinetic energy, speed and acceleration) between two flare-associated CME types. We investigated the relation between the CMEs kinetic energy and flare flux. The correlation coefficient for this relation is $R=0.40$, which is considered as weak relation.
\end{abstract}

Key words: Sun: flares - Sun: coronal mass ejections

\section{INTRODUCTION}

Solar flares and coronal mass ejections are two important phenomenaof the solar activity. A solar flare is a sudden flash of electromagnetic radiation emitted from the heated plasma and it is a giant explosion on the Sun's surface that occur when twisted magnetic field lines suddenly snap and release massive amounts of energy. Solar flares are rarely seen in the white light at photospheric region but easily visible in the chromospheric region at different wavelength bands (i.e. EUV and Xray). A coronal mass ejection is an eruption of magnetized plasma from the Sun into interplanetary space, and the phenomena of flare-associated CMEs have been observed during a solar prominence eruption (Munro et al. 1979).

The extrapolated CME onsets are determined as the time from first-order linear or second-order polynomials fit (plot) to the CME height-time data and the CME onset time backextrapolated to the flare site. For example is the flare-CME association event on 7 March 2012, an X5.4 flare started at 00:02 UT, peaked at 00:24 UT, flare location was

N18-E31, the heliocentric distance of the flare site was $0.585 \mathrm{R}_{\text {Sun }}$ and CME was observed by LASCO at 00:24 UT with a speed of 2684 $\mathrm{km} / \mathrm{s}$, then the extrapolated CME -onset is 00:15 UT at a height is $0.585 \mathrm{R}_{\text {Sun }}$ for the linear fit, this means that flare started 13 minutes earlier than the associated CME (i.e. BeforeCMEs), this time fall between the flare onset and maximum times.

Aarnio et al. (2011) have carried out the correlation analysis between flare flux and mass of CME using a distinct peak in the time separation distribution of time- and spatially constraint from 826 associated CME-flare events during the period 1996-2006 in cycle 23. They found a log linear relation-ship between the flare flux and CME mass. While Youssef (2012) has investigated the relationship between the flare-associated CME for temporally and spatially conditions using 776 CME-flare pairs during the time 1996-2010. He considered that the CME events were divided 
in two types, for investigating the correlation coefficient between the CME kinetic energy and flare flux. Compagnino et al. (2017) have studied the correlations between flares and CMEs properties based on a statistical analysis in the interval from 1997 to March 2014 over the solar cycles 23 and 24. Bidhu et al. (2017) examined the relation between the CMEs and flares properties in the maximum phases of 23 and 24 solar cycles. They detected larger numbers of CMEs in cycle 24 than the solar cycle 23 , although the cycle 24 were very weak, focusing on study of their speeds in the two cycles. More recently, Ibrahim et al. (2018) studied the CMEs and flares properties and flare-CME association using solar cycles 23 and 24, considering the CME data from the coordinated Data Analysis Workshops catalog and X-ray flares data from the Hinode / XRT satellite.

We presented an analysis of time- and spatially-correlated solar flares and CMEs, the statistical properties of these phenomena are important for our understanding of space weather. We studied the correlated properties of CME-flare in cycle 24, covering a time range of 2008-2017. A detailed analysis based on a more extended dataset including both of temporal and spatial relationships between flares and CMEs is reexamined in the period 2008-2017.

Our CME-flare events are spatially separating the sample into instances for distinguishing the effect of temporal and spatial datasets, which is clearly described as follows: CMEs that started before (After-CMEs) and after (Before-CMEs) the associated flares. We also show statistical distributions for the acceleration and velocity of CMEs with a choice of time interval of $60 \mathrm{~min}$. The dataset description is found in Section 2, while CMEs and flares properties are presented in Section 3. Summary of our main results and conclusions are given in Section 4.

\section{DATA DESCRIPTION}

In the following describe the procedure of selecting CME-flare associations data used in this analysis. We selected a sample of 519 flare-CME pairs in the period 2008-2017 over the cycle 24, where the associated CME was observed with the Large Angle Spectrometric Coronagraph (LASCO, Brueckner et al. 1995) instruments $\mathrm{C} 2$ and $\mathrm{C} 3$ on The Solar and Heliospheric Observatory (SOHO, Unzicker \& Donnelly 1974), which have a field of view of 2-6 and 6-30 solar radii, respectively. Based on the CME data set, there are 14,646 CMEs recorded in the period time 2008-2017, which provided by the Coordinated Data Analysis Workshops (CDAW, Gopalswamy et al. 2009) Data Center. While the solar flares datasets $(14,247$ events) are taken from the Hinode /XRT Satellite in the same period. As the temporal criterion, we used the \pm 60 -min time interval between the flare onset and CME on the field of view of the LASCO C2 coronagraph. We used the spatial constraint, following relation developed by Youssef (2012), in which the difference between the angular positions of the flare and associated CME in the field of the LASCO C2 coronagraph should be less than the angular size of the CME. The flare position angle, $\Psi_{\mathrm{F}}$, is given as a function of heliographic position from the longitude $(\lambda)$ and latitude $(\beta)$ (in degrees) of the flares locations, which can be calculated from the simplified expression (Youssef 2012; Isavnin 2014; Papaioannou et al. 2016):

$\psi_{\mathrm{F}}=\tan ^{-1}[\sin \lambda / \tan \beta]$

The spatial criterion used for selecting a flare-associated CME is defined according to this formula:

$\left|\psi_{\mathrm{CME}^{-}} \psi_{\mathrm{F}}\right|<\phi$

Where $\phi$ is the angular width (in degree) of CMEs, which refers to their projected locations in the plane of the sky. The CME position angle, $\psi_{\mathrm{CME}}$, and CME angular width $(\phi)$ are tabulated in the CDAW catalog of CMEs. The CME position angle is measured from Solar North (in degrees) in the counter-clockwise direction. The CME angular width is in the range from $\sim 2^{\circ}$ to $360^{\circ}$ (Yashiro et al. 2008). 
Using the spatial criterion, the flare position angle was determined from the heliographic position of the flare.

The mixture of both criteria directed to the creation of a sample of 519 CMEs-flares pairs that satisfied to both conditions. Next, we explained the algorithm that proposed by Youssef (2012), as the spatial condition. The CME time as found in the CDAW is that time when the CME is observed to enter the $\mathrm{C} 2$ or C3 instrument field of view, 1.5 or 3.7 solar radii from the center of the solar disk, respectively. We adopted the height-time data available from the CDAW catalog of CMEs for calculating onset times of the associated CMEflare. We estimated onset times of CMEs from the quadratic fit to height-time plots as given by (Gopalswamy et al., 2009).

\section{RESULTS OVER THE SOLAR CYCLE 24}

\subsection{The acceleration and speed distributions}

We have compiled a list of 519 flares occurring within $\pm 60 \mathrm{~min}$ from the onset of
CMEs for the period from December 2008 to April 2017. Figure 1 shows the acceleration distributions in our sample for all events (a). The average and median acceleration of the all CMEs are $\quad-5.0 \quad \mathrm{~m} \mathrm{~s}^{-2}$ and $-3.8 \quad \mathrm{~m} \mathrm{~s}^{-2}$, respectively. Also the mean and median acceleration for the After-CMEs (b) are $-6.5 \mathrm{~m}$ $\mathrm{s}^{-2}$ and $-5.1 \mathrm{~m} \mathrm{~s}^{-2}$, respectively. These events are clearly decelerated, hence these are poorly related to the flares; while the average and median acceleration for the Before-CMEs (c) are $-0.6 \mathrm{~m} \mathrm{~s}^{-2}$ and $0.3 \mathrm{~m} \mathrm{~s}^{-2}$, respectively, tend to be decelerated too, and are moderately related to associated flares, which depends on the difference between velocities of CMEs. The average acceleration of CMEs associated with flares of (27) B, (337) C-, (129) M- and (26) Xclass are $2.65 \mathrm{~m} \mathrm{~s}^{-2},-3.78 \mathrm{~m} \mathrm{~s}^{-2,}-4.38 \mathrm{~m} \mathrm{~s}^{-2}$ and $-28.6 \mathrm{~m} \mathrm{~s}^{-2}$, respectively.

Aarnio et al. (2011) investigated the associated CMEs with flares in the period 1996-2006 during cycle 23, for 826 flaresCMEs pairs. They found that the associated
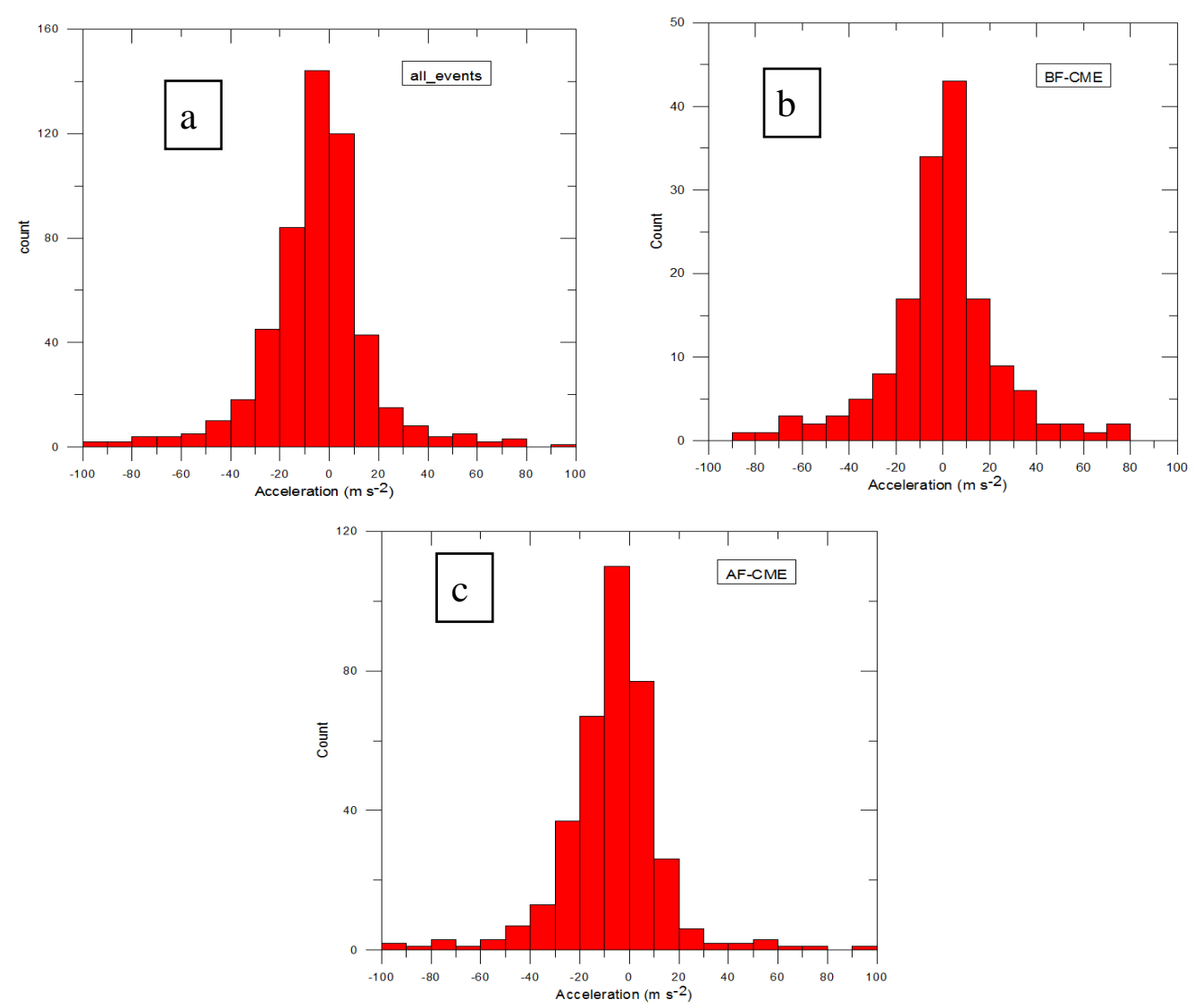

Figure 1: The acceleration distribution of the all events (a), the Before-CMEs (b) and the After-CMEs (c). 
CMEs with flares have negative mean acceleration of $-1.8 \mathrm{~m} \mathrm{~s}^{-2}$. Mickalek (2009) examined CMEs-flares in the period from 2000- 2004 of two types of CMEs associated flares for 578 events and found that first type AF-CMEs are decelerated, where (acc. $=-5.0 \mathrm{~m}$ $\mathrm{s}^{-2}$ ) and second type BF-CMEs are accelerated, where $\left(\right.$ acc. $\left.=5.0 \mathrm{~m} \mathrm{~s}^{-2}\right)$.

Next, we discuss the velocity distribution in the two CMEs samples. The velocity distributions in all events (a), Before-CMEs (b) and After-CMEs (c) are presented in Figure 2. The velocities in all events show mean speed of $633 \mathrm{~km} \mathrm{~s}^{-1}$ (median speed $=538 \mathrm{~km} \mathrm{~s}^{-1}$ ). The After-CMEs mean velocity is $528 \mathrm{~km} \mathrm{~s}^{-1}$. On the other hand, the Before-CMEs have higher mean velocity of $\left(870 \mathrm{~km} \mathrm{~s}^{-1}\right)$. We noted that the Before-CMEs has a faster velocity (median speed $=725 \mathrm{~km} / \mathrm{s}$ ) as compared to the AfterCMEs which is slightly slower (median speed $=$ $441 \mathrm{~km} / \mathrm{s}$ ). Also, we found the average speeds of the all events and After-CMEs are smaller



than Before-CMEs associated with flares for our sample. A clear and important difference between velocities in the two samples of CMEs, i.e. a large difference is noticeable between both types. The differences in the velocities between both categories allow us to explain the dynamics of the acceleration behavior of CMEs by drag.

We collected our data set of the associated flares with CMEs in a time window of $\pm 60 \mathrm{~min}$. We present 519 flares and CMEs pairs that are temporally and spatially associated in the period of 2008-2017. The height-time data was used to correct for the CMEs onsets time. We included the CMEs-flares distributions on a time separation of $\sim 60 \mathrm{~min}$; due to the CMEs and flares events are spatially correlated and represent a valid time separation in this period. The mean difference in time separation $(\Delta \mathrm{T})$ between the initiation time of CMEs and flares was $(<\Delta \mathrm{T}\rangle=-13 \mathrm{~min})$. Figure 3 shows the difference time $(\Delta \mathrm{T})$ separation between both

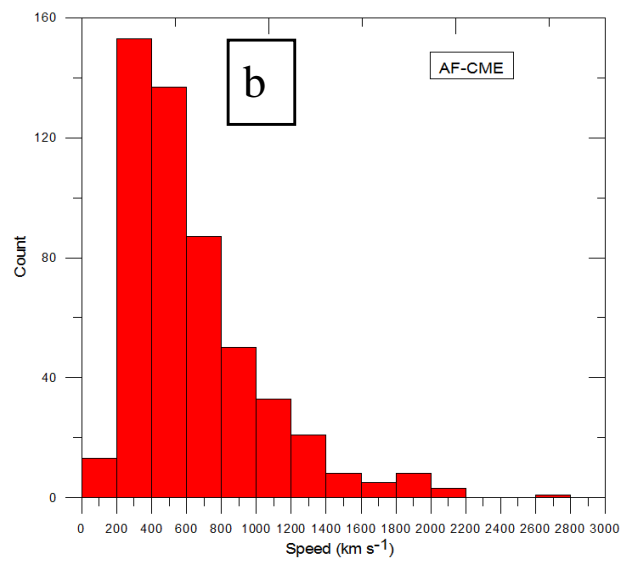

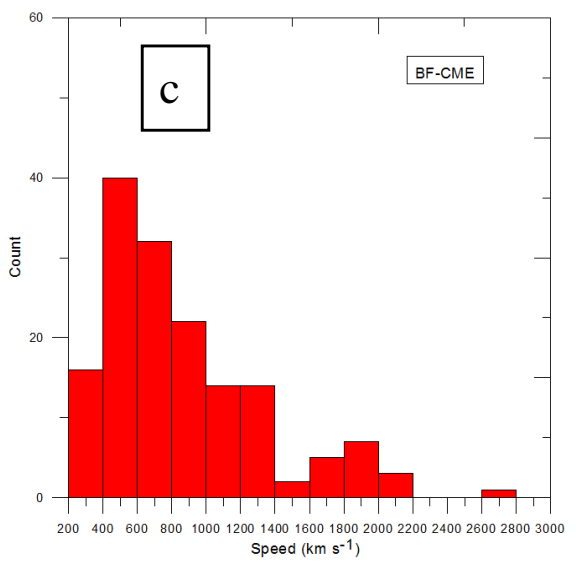

Figure 2: The speed distributions of the all (a), AF-CMEs (b) and the BF-CMEs (c). 
events over all the sample. The CMEs and flares time separation represent almost symmetrical and Gaussian distributions. We noted that most of the associated CMEs with flares have a time separation lower than \pm 30 $\min$.

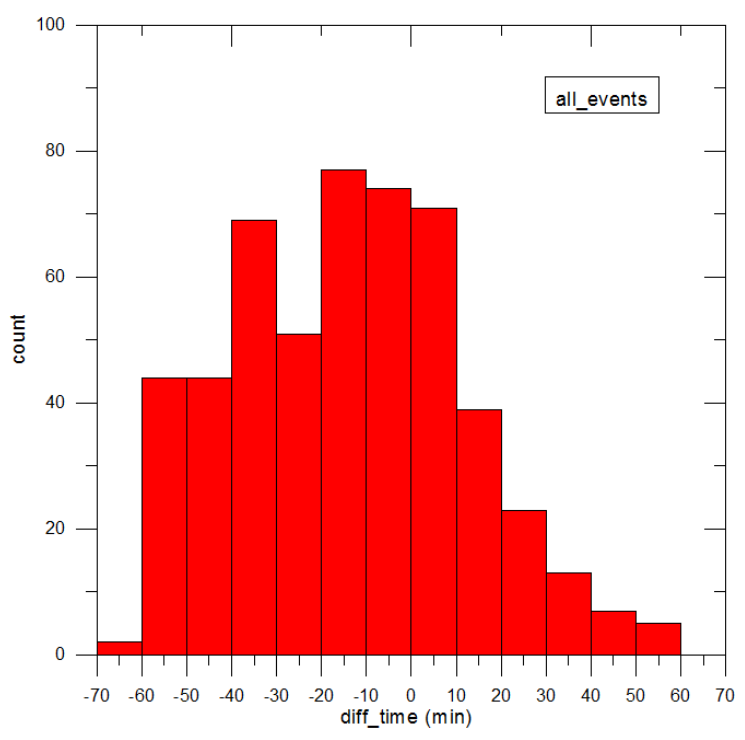

Figure 3: The distribution of the difference times between the onset time of CMEs and flares with a time window $\pm 60 \mathrm{~min}(\Delta \mathrm{T}=\mathrm{CME}$ onset time flare onset time).

\subsection{Distribution of flare class associated with CME in our sample}

The flare classes in our sample (519) events are $\mathrm{B}, \mathrm{C}, \mathrm{M}$ and $\mathrm{X}$. The number of each flare class and its percent is given in Table 1 and shown in Fig 4. The majority of the sample is in C-class. The ascending order of the flare class is $\mathrm{X}, \mathrm{B}, \mathrm{M}$ and $\mathrm{C}$.

Table 1. The distribution of all, After- and Before-CMEs events have B-, C-, M-, and $\mathrm{X}$-class flares in the period 20082017.

\begin{tabular}{|c|c|c|c|}
\hline Flare-class & $\begin{array}{c}\text { No. of All- } \\
\text { CMEs }\end{array}$ & $\begin{array}{c}\text { No. of After- } \\
\text { CMEs }\end{array}$ & $\begin{array}{c}\text { No. of } \\
\text { Before-CMEs }\end{array}$ \\
\hline B & $27(5.20 \%)$ & $18(3.47 \%)$ & $9(0.01 \%)$ \\
\hline C & $337(65 \%)$ & $251(48.36 \%)$ & $86(16.57 \%)$ \\
\hline M & $129(24.9 \%)$ & $77(14.84 \%)$ & $52(10.01 \%)$ \\
\hline X & $26(5 \%)$ & $13(2.50 \%)$ & $13(2.50 \%)$ \\
\hline
\end{tabular}

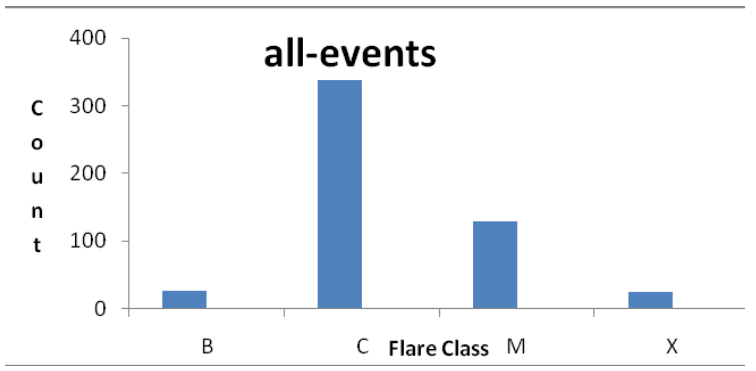

Figure 4: Distribution of flare-class associated with CME for all samples.

\subsection{The CME energy and flare flux correlation}

We investigated the relationship between the energy of CME and peak flux of flare for both CME categories in the CMEs associated flares sample. The correlation coefficient between CME energy and flare flux is $\mathrm{R}=0.40$ for all the sample. Hence the correlation between them is a weak one, the After-CMEs shows a weak linear Pearson correlation coefficient of $(\mathrm{R}=0.37)$ with an interesting result in the Before-CMEs which reveals a slightly larger correlation $(\mathrm{R}=0.55)$ than the After-CMEs. Weak correlations are obtained from the CME energy and flare flux in both CMEs samples. The After-CMEs mean kinetic energy is $1.33 \mathrm{E}^{+31} \mathrm{erg}$. But the mean kinetic energy is $5.39 \mathrm{E}^{+31} \mathrm{erg}$ for the Before-CMEs. We notice that there are clear smaller difference in the average energies between both CMEs categories. We also compare the median energies in the two CMEs samples, and found difference in the energy. The Before-CMEs (a median energy $=1.1 \mathrm{E}^{+31} \mathrm{erg}$ ) is systematically more energetic than the After-CMEs (a median energy $=1.2 \mathrm{E}^{+30} \mathrm{erg}$ ). There is one order of magnitude difference, we also compared the median energies in the two CMEs samples; we found that the Before-CMEs is higher energies than After-CMEs. Youssef (2012) realized different correlations between the CME energy and flare flux in the period 1996-2010, who involved $776 \mathrm{CME}-$ flare events. $\mathrm{He}$ also investigated the two CMEs patterns with applying the temporal method in a time window of $\pm 60 \mathrm{~min}$, while the spatial criterion was the same used in this study. The BeforeCMEs provided a correlation of (0.68), while the correlation for the After-CMEs sample was (0.46). These correlations are higher than those obtained in our study. 

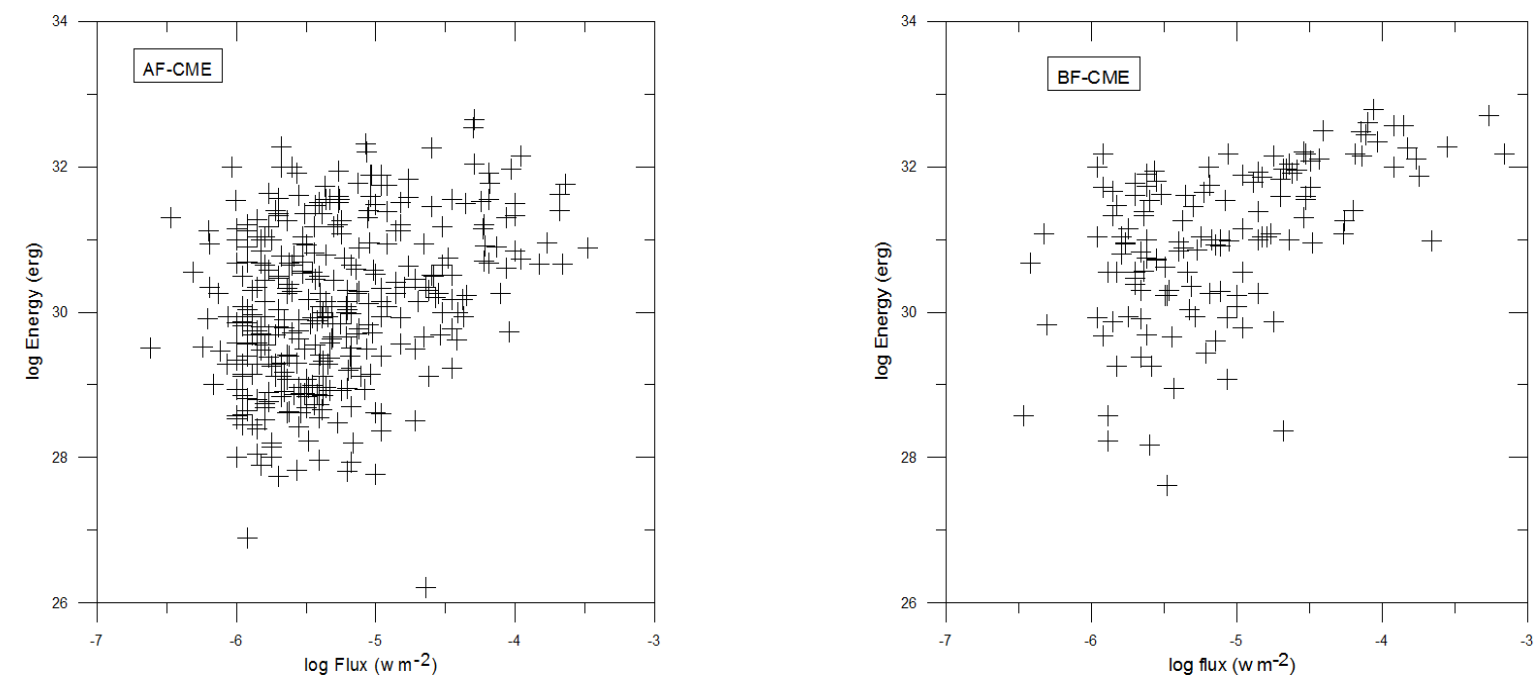

Figure 5: CME-Flare correlation for After-CMEs (left-panel) and Before-CMEs (right-panel).

\section{DISCUSSION AND CONCLUSION}

We have studied the relation between the coronal mass ejection (CME) and the solar flare during solar cycle 24 from December, 2008 to April, 2017. It has been shown that the CME associated with flare for our sample is 519 events, using the temporal and spatial criteria for the selected CME-Flare events with a time window $\pm 60 \mathrm{~min}$ of the CME-categories (After-CME and Before-CME). We studied the correlation between the CME energy and flare flux for all, After-CMEs and Before-CMEs. It is noticed that the correlation coefficient between the energy of CME and X-ray flare flux is $R=0.41$ for all events, which show a weak relation. Also, we studied two types of CME associated with flares (After-CMEs and Before-CMEs), and found that the correlation coefficient between flare flux and CME energy is $\mathrm{R}=0.33$ poor correlation for After-CMEs and $\mathrm{R}=0.54$ is found which show better correlate for Before-CMEs.

We present an analysis of the correlated solar flares and coronal mass ejections acquiring both of time and spatially constraints, using a time range of 2008-2017 during the cycle 24 . We studied the physical properties of coincident flares and CMEs in which a flare preceded a CME (Before-CMEs) and a flare followed a CME (After-CMEs) during this period. The acceleration distributions of all
CMEs events are clearly decelerated (mean acceleration $=-5.0 \mathrm{~m} \mathrm{~s}^{-2}$ ) associated to $\mathrm{a}$ slightly fast speed $($ mean speed $=633 \mathrm{~km} / \mathrm{s}$ ). We first compared the physical properties of coincident flares and CMEs for a flare that started after the CME (After-CMEs) event. The flares followed the associated CMEs are clearly decelerated (mean acceleration $=-6.5 \mathrm{~m} \mathrm{~s}^{-2}$ ). These events are significantly induced by a slower velocity (mean speed $=530 \mathrm{~km} / \mathrm{s}$ ).

This CMEs type is poorly correlated to the coincident flares, where the correlation coefficient between the logarithms of the CME energy and flare flux shows a weak linear Pearson correlation coefficient of $(r=0.37)$. On the other hand, the flares preceded the associated CMEs are slightly accelerated (median acceleration $=0.4 \mathrm{~m} \mathrm{~s}^{-2}$ ). This type is characterized with a faster velocities (median speed $=721 \mathrm{~km} / \mathrm{s}$ ). The Before-CMEs is moderately associated to the flares, the logarithms of the CME energy and flare flux indicates amoderate Pearson correlation coefficient of $(r=0.55)$.

We concluded that the Before-CMEs show the same correlation resulting from the two correlations analyses. The After-CMEs show a linear Pearson correlation of $\sim 0.65$, while the Before-CMEs displays a correlation of $\sim 0.53$ between both parameters. Michalek (2009) found out that the After- and Before-CMEs in 
cycle 23 were shown to give a weak correlations to the associated flares by $\sim 0.44$ and $\sim 0.30$, respectively. TheAfter- and BeforeCMEs show similar distributions of CMEs that associated with the four B-, C-, M-, and X-class flares (Figure 4). We identify the correlated properties of time- and spatially-correlated flares and CMEs during this period, in which a flare preceded a Before-CMEs versus when one followed After-CMEs events which differ in their physical properties, indicating potentially different initiation mechanisms.

\section{REFERENCES}

Aarnio A.N., Stassun K.G., Hughes W.J. and McGregor S.L. 2011, Solar Phys. 268, 195.

Bak-Steslicka U., Kolomanski S., Mrozek T. 2013, Coronal mass ejection associated with slowing duration flares. Sol. Phys. 283, 505-517.

Bidhu SS, Sobia AI and Benjamin D. 2017, J Space Explor., 6, 122, "CME Speed and Angular Width Distributions during 23 and 24 Solar Cycle Maximum"

Brueckner G.E. et al. 1995, Solar Phys., 162, 357

Compagnino A., Romano P., and Zuccarello F. 2017, Solar Phys., 292, 5

Gopalswamy N., Akiyama S., and Yashiro S., 2009, IAUS, 257, 283
Ibrahim M. S., Shanmugaraju A., Moon Y.-J., Vrsnak B., and Umapathy S., Advances in Space Research 61 (2018) 540-551

Isavnin, A. 2014, PhD, "Flux ropes in space plasmas", University of Helsinki Michalek G. 2009, A \&A, 494, 263-268

Munro R. H., Gosling J. T., Hildner E., et al. 1979, Sol. Phys., 61, 201

Papaioannou A., Sandberg I., Anastasiadis A. and Kouloumvakos A., Georgoulis M. K., Tziotziou K., Tsiropoula G., Jiggens P., Hilgers A. 2016, J. Space Weather Space Clim., 6, A42, DOI: $10.1051 / \mathrm{swsc} / 2016035$

Unzicker, A., and R.F. Donnelly, 1974, Calibration of X-ray ion chambers for the Space Environment Monitoring System. Technical report COM-75-10667. In: National Oceanic and Atmospheric Administration, Boulder, Colo. (USA). Space Environment Lab

Youssef M. 2012, "On the relation between the CMEs and the solar flares", NRIAG Journal of Astronomy and Geophysics, 1, 172-178, DOI: 10.1016/j.nrjag.2012.12.014.

Yashiro S., Gopalswamy N., Michalek G., et al. 2004, J. Geophys. Res., 109, Cite ID A07105

Yashiro S., Michalek G., Akiyama S., Gopalswamy N. and Howard R. A., 2008, ApJ, 673, 1174 